\title{
Individual and combined associations between cardiorespiratory fitness and grip strength with common mental disorders: a prospective cohort study in the UK Biobank
}

Aaron A. Kandola ${ }^{1 *}$ D, David P. J. Osborn ${ }^{1,2}$, Brendon Stubbs ${ }^{3,4}$, Karmel W. Choi ${ }^{5,6,7,8}$ and Joseph F. Hayes ${ }^{1,2}$

\begin{abstract}
Background: Depression and anxiety are common mental disorders that increase physical health risks and are leading causes of global disability. Several forms of physical fitness could be modifiable risk factors for common mental disorders in the population. We examined associations between individual and combined markers of cardiorespiratory fitness and grip strength with the incidence of common mental disorders.
\end{abstract}

Methods: A 7-year prospective cohort study in 152,978 UK Biobank participants. An exercise test and dynamometer were used to measure cardiorespiratory and grip strength, respectively. We used Patient Health Questionnaire-9 and Generalised Anxiety Disorder-7 scales to estimate the incidence of common mental disorders at follow-up.

Results: Fully adjusted, longitudinal models indicated a dose-response relationship. Low and medium cardiorespiratory fitness was associated with 1.485 (95\% Cls, 1.301 to $1.694, p<0.001)$ and 1.141 (95\% Cls, 1.005 to $1.297, p=0.041$ ) higher odds of depression or anxiety, compared to high cardiorespiratory fitness. Low and medium grip strength was associated with 1.381 ( $95 \% \mathrm{Cls}, 1.315$ to $1.452, p<0.001)$ and 1.116 (95\% Cls, 1.063 to 1.172 , $p<0.001$ ) higher odds of common mental disorder compared to high grip strength. Individuals in the lowest group for both cardiorespiratory fitness and grip strength had 1.981 ( $95 \%$ Cls, 1.553 to 2.527, $p<0.001$ ) higher odds of depression, 1.599 (95\% Cls, 1.148 to $2.118, p=0.004$ ) higher odds of anxiety, and 1.814 ( $95 \%$ Cls, 1.461 to $2.252, p<0.001)$ higher odds of either common mental disorder, compared to high for both types of fitness.

Conclusions: Objective cardiorespiratory and muscular fitness markers represent modifiable risk factors for common mental disorders. Public health strategies to reduce common mental disorders could include combinations of aerobic and resistance activities.

Keywords: Fitness, Depression, Anxiety, Physical activity, Exercise, Cardiorespiratory, Grip strength, Muscular, Prevention

\footnotetext{
* Correspondence: a.kandola.18@ucl.ac.uk

'Division of Psychiatry, University College London, Maple House, 149 Tottenham Court Rd, London W1T 7BN, UK

Full list of author information is available at the end of the article
}

C C The Author(s). 2020 Open Access This article is licensed under a Creative Commons Attribution 4.0 International License, which permits use, sharing, adaptation, distribution and reproduction in any medium or format, as long as you give appropriate credit to the original author(s) and the source, provide a link to the Creative Commons licence, and indicate if changes were made. The images or other third party material in this article are included in the article's Creative Commons licence, unless indicated otherwise in a credit line to the material. If material is not included in the article's Creative Commons licence and your intended use is not permitted by statutory regulation or exceeds the permitted use, you will need to obtain permission directly from the copyright holder. To view a copy of this licence, visit http://creativecommons.org/licenses/by/4.0/. The Creative Commons Public Domain Dedication waiver (http://creativecommons.org/publicdomain/zero/1.0/) applies to the data made available in this article, unless otherwise stated in a credit line to the data. 


\section{Background}

Common mental disorders are major contributors to the global health burden, with depression and anxiety disorders being the first and sixth leading causes of disability worldwide, respectively [1]. They can substantially affect daily functioning and are associated with elevated physical health risks over time, including a higher incidence of cardiovascular disease and premature mortality [2-4]. Structured physical activity interventions have been shown to reduce common mental health symptoms with a moderate-to-large effect size [5-11]. Low physical activity may be a modifiable, population-level risk factor for common mental disorders [12-16]. However, nearly prior all studies use self-reported measures of activity, which are prone to bias [17]. Physical activity has a major influence on cardiorespiratory fitness (CRF) and muscular strength $[18,19]$, two related but distinct markers of physical fitness that are reliable indicators of overall health, disease risk, and mortality [20-27]. Both are measurable in large groups through validated fitness tests that produce objective outputs, which may also act as surrogate markers of habitual physical activity that are not reliant on self-report $[18,28]$.

There is limited research on the associations between physical fitness markers and the incidence of common mental disorders. A recent meta-analysis of prospective cohort studies found that low CRF was associated with a $47 \%(\mathrm{HR}=1.47,95 \% \mathrm{CI} 1.23,1.76)$ higher incidence rate of common mental disorders [28]. However, these results were from a small number of studies $(n=4)$ with substantial heterogeneity $\left(I^{2}=85.1 \%\right)$ and most focused on depression rather than anxiety. Fewer studies have focused on the role of overall muscular strength, for which grip strength is a simple clinical proxy measure [29-31]. Some small cross-sectional [32, 33] and longitudinal [34-36] studies suggest that low grip strength is associated with a higher incidence of depression and one with anxiety [37]. However, longitudinal findings have been inconsistent, and more high-quality data from large samples are necessary. Most studies only focus on depression, despite anxiety disorders being another major source of global disability and highly comorbid with depression [38].

Low physical fitness, indexed by CRF or grip strength, could be a useful risk factor for common mental disorders in the population. We are not aware of any largescale studies focusing on associations between individual and combined CRF and grip strength with the incidence of common mental disorders. CRF and grip strength reflect different physiological profiles and indicate different types of habitual physical activity, i.e. aerobic vs. resistance training. Combined training to increase both CRF and strength leads to improved physical health outcomes than focusing on either component of fitness alone [39-42] and the same may be true for mental health. A recent cross-sectional study in adolescents found that CRF and not muscular strength was associated with fewer psychological difficulties, but did not assess the combination of CRF and muscular strength [43].

To address these knowledge gaps, we conducted the first prospective study to examine associations between individual and combined markers of CRF and grip strength with the incidence of depression and anxiety. We aimed to (1) examine longitudinal associations between CRF, grip strength, and the incidence of common mental disorders and (2) investigate associations between combined fitness levels with the incidence of common mental disorders.

\section{Methods}

\section{Participants}

The UK Biobank is a prospective cohort study that recruited 502,682 participants (5.5\% response rate) aged 40 to 69 years from the general population of England, Scotland, and Wales, between April 2007 and December 2010 [44]. Participants completed a baseline battery of touchscreen questionnaires, physical measures, imaging, genetic, and biological assessments in 22 research centres across the UK [45]. Our sample included participants who had a valid measure of baseline symptoms and at least one measure of grip strength or CRF ( $n=$ $491,278)$ at baseline. For the longitudinal analyses, we restricted the sample to participants who also had a completed Patient Health Questionnaire-9 (PHQ-9) and Generalised Anxiety Disorder-7 (GAD-7) at follow-up (2017) and at least one measure of grip strength or CRF $(n=152,978)$.

\section{Exposure(s): cardiorespiratory fitness and grip strength}

A subset of Biobank participants completed fitness tests between August 2009 and December 2010. Participants completed a 6-min submaximal exercise test on a stationary bike (eBike Comfort Ergometer, General Electric). Individualised protocols were developed to calculate an appropriate workload based on participants' age, height, weight, sex, and resting heart rate. A four-lead ECG was used to monitor heart rate before, during, and in the recovery phase following the test.

We followed protocols from previous studies to estimate CRF from the submaximal tests $[46,47]$. We first estimated the work rate participants would have achieved in a maximal fitness test. The maximal work rate maps onto maximum oxygen consumption $\left(\mathrm{VO}_{2 \max }\right)$, which is an indicator of CRF. We estimated maximal work rate (measured in watts) from heart rate before the test, maximum heart rate during the test, and work rate at the end of the test using linear regression. We then extrapolated the regression line to participants' 
age-predicted maximal heart rate using the equation: $208-7 \times$ age [48], assuming a linear relationship.

To estimate maximum oxygen consumption, we used the equation: $7+(10.8 \times$ maximal work rate (in watts) $) /$ body weight (in $\mathrm{kg}$ )) [49]. Maximal oxygen consumption is a continuous measure of CRF expressed in millilitres of oxygen per kilogramme of body weight per minute $(\mathrm{ml}$ kg-1.min-1). We used metabolic equivalents (METs) to express CRF output, where 1 MET is $3.5 \mathrm{ml} \mathrm{kg-1.min-1.}$

Grip strength was measured using a Jamar j00105 hydraulic hand dynamometer in each hand. Participants grasp the handle sitting in an upright position and squeeze as strongly as possible for $3 \mathrm{~s}$. The output was expressed in kilogrammes $(\mathrm{kg})$, taking the mean value of each hand.

For the main analysis, we created age- and sexadjusted tertiles of grip strength and CRF, in line with previous work [47]. The tertiles represent low, medium, and high fitness groups. These groupings were to aid interpretation and account for possible non-linear associations between fitness and mental health. We also used continuous CRF and grip strength exposure variables in the secondary analysis, with CRF presented in METs and grip strength in 5 $\mathrm{kg}$ increments as in previous studies [46].

\section{Outcome(s): common mental disorders}

At baseline (2006 to 2010), common mental health symptoms were measured using 3 questions from $\mathrm{Pa}$ tient Health Questionnaire-9 (PHQ-9) that cover core features of depression (low mood, anhedonia, and lethargy) [50]. It contains an additional question adapted from the PHQ-9 to cover tenseness, a common feature of anxiety disorders. The questionnaire uses a four-point ordinal scale from 0 (not at all) to 3 (nearly every day), with scores ranging from 0 to 12 . Ultra-brief adaptations of the PHQ-9 have good agreement with longer scales for both depression and anxiety symptoms [51]. We used a continuous symptom score for this measure due to the lack of a valid cut-off.

At follow-up, common mental health symptoms were measured in a 2017 Mental Health Questionnaire, which included the PHQ-9 and Generalised Anxiety Disorder-7 (GAD-7) questionnaire. The PHQ9 is a depression screening instrument with nine questions on a four-point ordinal scale from 0 (not at all) to 3 (nearly every day) [50]. Total scores range from 0 to 27, with higher scores indicating greater symptom severity. We defined possible incidences of depression using an established cut-off (scores $\geq 10$ ) for the main analysis and a continuous symptom score (secondary analysis). Previous studies show that a cut-off score of $\geq 10$ has $88 \%$ sensitivity and specificity for identifying incident major depression [50].
The GAD-7 is a 7-item anxiety scale using the same four-point ordinal scale as the PHQ-9 [52]. Scores range from 0 to 21 . We defined possible incidences of generalised anxiety disorders using an established cut-off (scores $\geq 10$ ) in the main analysis and continuous symptom score for the secondary analysis. This cut-off has a sensitivity of $89 \%$ and specificity of $82 \%$ [52].

\section{Confounding variables}

We constructed a directed acyclic graph (DAG) of the proposed causal assumptions between exposure, outcome, and confounding variables that informed our analysis (Additional file 1, Figure 1). Possible confounding variables for this analysis include the following: age, sex, socioeconomic position (household income of $<£ 18,000$, $£ 18,000$ to $£ 30,999, £ 31,000$ to $£ 51,999, £ 52,000$ to $£ 100$, 000 , and $>£ 100,0000)$, baseline mental health symptoms, smoking status (current, former, or never), total physical activity (total daily minutes spent walking and in moderate or vigorous activity from the International Physical Activity Questionnaire (IPAQ)), education level (degree, A/AS-level, O-level/GCSE, CSE, NVQ/HND/HNC, other qualifications, none), parental depression, chronic illness (self-reported yes or no), and diet (pieces of fruit and vegetable per day).

Our DAG indicated that adiposity (body fat percentage) could be on the causal pathway between fitness and common mental health symptoms. To avoid overadjustment, we only include body fat as a confounding variable in the sensitivity analysis. Other variables used in the sensitivity analyses include a self-reported indicator of having visited a doctor or psychiatrist for depression, nerves, or anxiety in the past to identify participants with a history of common mental disorders.

\section{Analysis}

Descriptive variables include means and standard deviations for normally distributed variables and medians and interquartile ranges for non-normally distributed variables.

\section{Main analysis}

The main analysis consists of two main components. The first aimed to determine longitudinal associations between individual domains of CRF, grip strength, and common mental health symptoms and disorders (aim 1). The second part investigated longitudinal associations between combined fitness and the incidence of common mental disorders (aim 2).

Firstly, we used logistic regression models with depression and anxiety incidence as outcomes and grip strength and CRF as categorical exposures, in separate models. We ran crude and fully adjusted iterations of all models. Secondly, the analysis of the combined role of 
CRF and grip strength derived logistic regression models with the same mental health outcomes and adjustments as our initial longitudinal models but using combined fitness as the main categorical exposure. Combined fitness includes both CRF and grip strength, which were positively correlated $(r=0.40)$. We compared combinations of CRF and grip strength, using the high CRF and high grip strength group as the reference category.

\section{Secondary and sensitivity analysis}

In the secondary analysis, we examined cross-sectional associations between CRF and grip strength with common mental health symptoms at baseline $(n=491,278)$. We used negative binomial regression models as with symptom scores on a quasi-continuous scale. We used negative binomial regression to account for the high positive skew and over-dispersion of the symptom scores. We present the output of negative binomial regressions as a percentage change in symptom scores. High fitness was the reference category for both exposure models.

We also reran the first part of the main analysis using continuous exposure variables (per 1-MET for CRF and per $5 \mathrm{~kg}$ for grip strength). We then created age and sexstandardised $\mathrm{z}$ scores for each exposure and ran the same models with age- and sex-exposure as multiplicative interaction terms to examine possible interactions.

We also carried out several sensitivity analyses determined a priori to examine the robustness of our main findings and explore alternative explanations. These included rerunning the longitudinal models from part 1 of the main analysis and in separate models: (1) excluding participants with a self-reported history of depression or anxiety prior to the baseline to further reduce the risk of residual confounding from reverse causation, (2) including adiposity as a possible confounding variable to examine the alternative hypothesis that adiposity is not on the causal pathway, and (3) using lower thresholds for defining depression (PHQ $\geq 8$ ) and anxiety (GAD-7 $\geq 8$ ) disorders $[53,54]$.

We then performed an adjusted multivariate linear model with CRF and grip strength as continuous exposures and PHQ-9 and GAD-7 scores as continuous outcomes. This was to assess whether both exposures were associated with depression and anxiety outcomes independently by testing each exposure coefficient across the equations for both outcomes. We also calculated $e$ values to assess the plausibility of unmeasured confounding affecting our findings [55]. The $e$ value estimates the required strength of an unmeasured confounding variable that would nullify the observed associations between our exposure and outcomes, while accounting for all measured covariates [56].
We reran the first part of the main longitudinal analysis in a full cohort with imputed missing data to assess the risk selection bias within our longitudinal sample $(n=152,978)$. We used multiple imputation models with chained equations to estimate missing data, as detailed in Methodology 1 (Additional file 1).

\section{Results \\ Participants}

At baseline, there were 491,278 participants with complete CRF or grip strength data. For the adjusted longitudinal (main) analysis, 147,141 participants had complete data for grip strength and 22,667 for CRF. For the cross-sectional (secondary) analysis, there were 465, 757 participants in the fully adjusted analysis for grip strength and 60,838 for CRF data. According to the PHQ-9 and GAD-7 scales, 9156 (5.99\%) participants met the criteria for depression, 5282 (3.45\%) for anxiety, and 11,295 (7.39) for either common mental disorder at the 7-year follow-up. Tables 1 and 2 (Additional file 1) respectively contain baseline characteristics of participants by the CRF group and by grip strength group.

\section{Main analysis}

Longitudinal models for CRF and grip strength as separate exposures with incident depression, anxiety, and depression or anxiety as outcome variables are presented in Table 1. Adjusted models indicate that low CRF was associated with a $1.596(95 \%$ CIs, 1.378, $1.849 p<0.001)$ and medium CRF was associated with a 1.154 (95\% CIs, $0.999,1.334, p=0.051)$ increase in the odds of depression compared with the high CRF group. Low CRF was associated with a 1.230 (95\% CIs, 1.020, 1.483, $p=0.030)$ increase in the odds of anxiety compared to high CRF. Low CRF was associated with a 1.485 (95\% CIs, 1.301, 1.694, $p<0.001)$ and medium CRF with a $1.141(95 \%$ CIs, 1.005, 1.297, $p=0.041$ ) increase in the odds of either common mental disorder compared to high CRF.

Low grip strength was associated with a 1.410 (95\% CIs, $1.355,1.490, p<0.001)$ and medium with a 1.126 (95\% CIs, 1.066, 1.189, $p<0.001$ ) increase in the odds of depression compared with high grip strength. Compared with high, low grip strength was associated with a 1.380 (95\% CIs, 1.286, 1.480, $p<0.001$ ) and medium with a 1.145 (95\% CIs, 1.068, 1.228, $p<0.001$ ) increase in the odds of anxiety. Low grip strength was associated with 1.381 (95\% CIs, 1.315, 1.452, $p<0.001$ ) and medium with $1.116 \%$ (95\% CIs, 1.063, 1.172, $p<0.001$ ) higher odds of depression or anxiety.

Table 2 shows the results from the combined fitness exposures of CRF and grip strength. Compared to the high fitness group (high CRF and grip strength), low fitness (low CRF and low strength) was associated with 1.981 (95\% CIs, 1.553, 2.527, $p<0.001$ ) and medium 


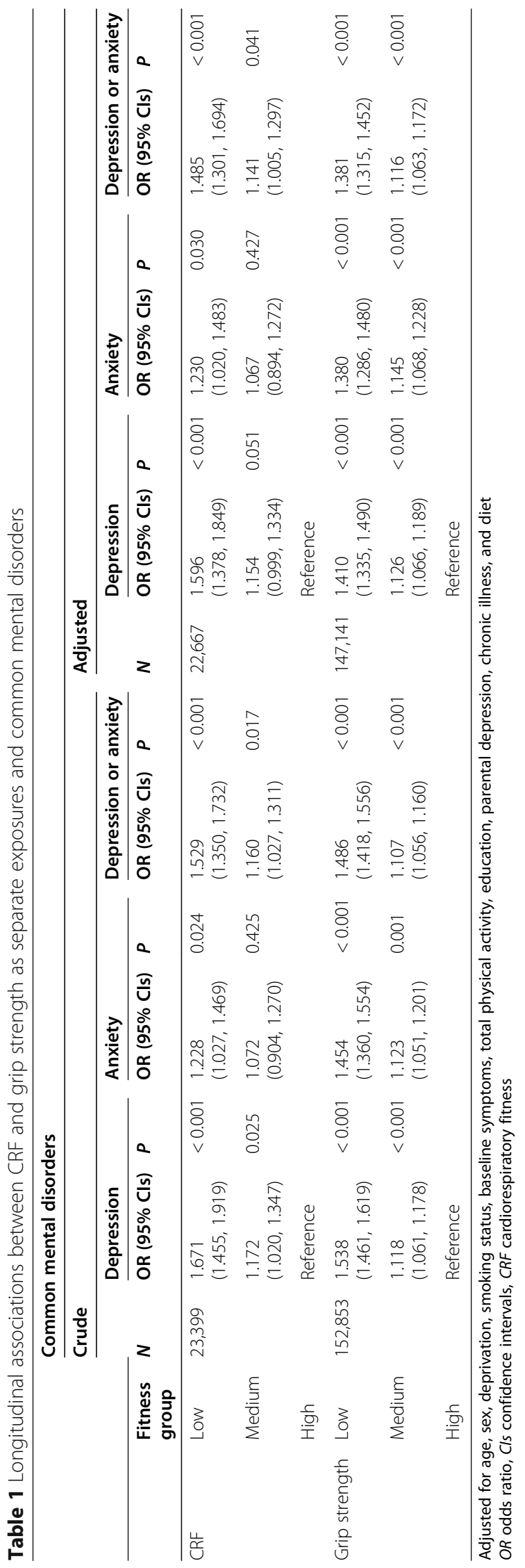




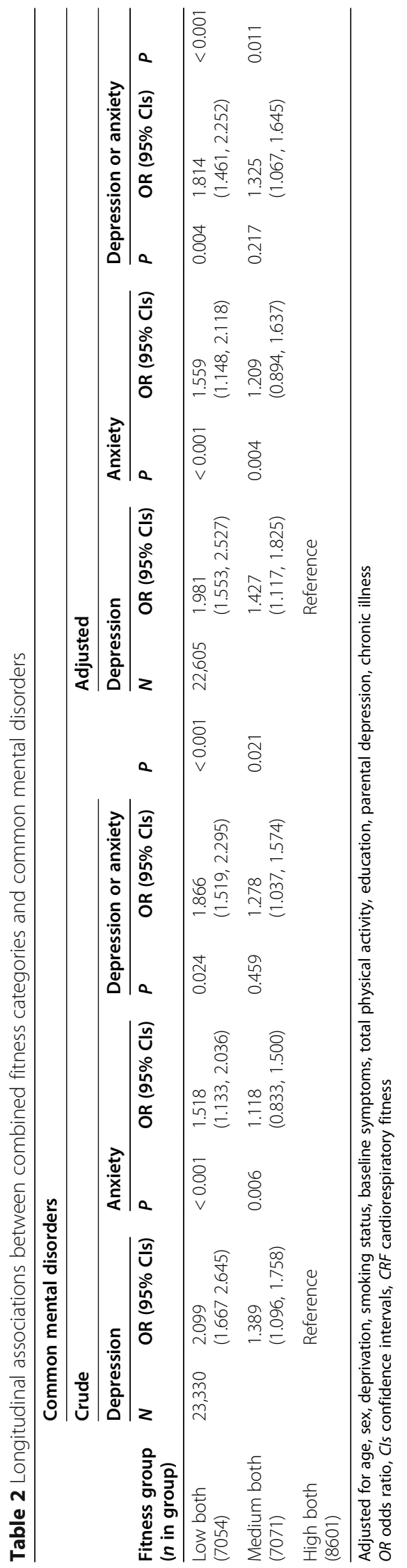


with 1.427 (95\% CIs, 1.117, 1.825, $p=0.004$ ) higher odds of depression. Low fitness was associated with 1.599 (95\% CIs, 1.148, 2.118, $p=0.004$ ) higher odds of an anxiety disorder, compared with high fitness. Low fitness was associated with a 1.814 (95\% CIs, 1.461, 2.252, $p<0.001$ ) and medium with a 1.325 (95\% CIs, 1.067, $1.645, p=0.011$ ) increase in the odds of depression or an anxiety disorder, compared with high combined fitness.

\section{Secondary analysis and sensitivity analysis}

The fully adjusted cross-sectional analysis examining associations between CRF and common mental health symptoms included 63,372 participants. The models suggest a biological gradient with the medium CRF group associated with a $7 \%$ higher symptom score $(95 \%$ CIs, $4.4 \%, 9.6 \%, p<0.001$ ) and low CRF associated with $17.5 \%$ higher symptom score (95\% CIs, $14.6 \%, 20.6 \%$, $p<0.001)$ compared to the high CRF group. A similar association was observed for grip strength: compared to the high grip strength group, the medium group had 8.6\% (95\% CIs, 7.7\%, 9.6\%, $p<0.001$ ) higher scores and the low group had $26.8 \%$ (95\% CIs, 25.7\%, 27.9\%, $p<0.001$ ) higher scores.

Fully adjusted longitudinal models with a continuous exposure suggest each 1 MET increase in CRF was associated with a $2.4 \%$ lower depression score $(95 \% \mathrm{CI},-3 \%$, $-1.8 \%, p<0.001)$ and a $1.22 \%$ lower anxiety score $(95 \%$ $\mathrm{CI},-2 \%,-0.3 \%, p<0.001)$. Each $5 \mathrm{~kg}$ increase in grip strength was associated with a $4.4 \%$ lower depression score $(95 \% \mathrm{CI},-4.8 \%,-3.9 \%, p<0.001)$ and a $4.8 \%$ lower anxiety score $(95 \% \mathrm{CI},-5.4 \%,-4.2 \%, p<0.001)$.

There was some evidence of an interaction between grip strength and sex $(p=0.018)$ and grip strength and age $(p=0.001)$ for anxiety disorders. For men, each $5 \mathrm{~kg}$ increase in grip strength was associated with reduced odds of an anxiety disorder (OR $=0.901,95 \% \mathrm{CI}, 0.855$, $0.950, p<0.001)$; for women, this reduction was greater $(\mathrm{OR}=0.840,95 \% \mathrm{CI}, 0.809,0.873, p<0.001)$. In those aged $<54$, a $5-\mathrm{kg}$ increase in grip strength was associated with $12.5 \%$ reduction in odds of anxiety $(\mathrm{OR}=0.875$, 95\% CI, 0.826, 0.927, $p<0.001)$, ages $\geq 54$ to 65 with $22.8 \%$ reduced odds $(\mathrm{OR}=0.772,95 \% \mathrm{CI}, 0.711,0.837$, $p<0.001)$, and those $\geq 65$ with $27.3 \%$ reduced odds $(\mathrm{OR}=0.727,95 \% \mathrm{CI}, 0.623,0.850, p<0.001)$.

The results of our sensitivity analyses (see Additional file 1, Tables 3 to 6) indicate no substantial differences from our main findings. The results from our analyses in a fully imputed cohort were consistent with the findings in the main analysis (Additional file 1, Table 6). The $e$ values estimate the required strength of an unmeasured confounding variable to nullify the observed associations between our exposure and outcomes. For longitudinal models of the association between low CRF (vs. high) with depression, the $e$ value was $2.57(\mathrm{CI}=2.1)$, with anxiety was $1.76(\mathrm{CI}=1.16)$, and depression or anxiety was $2.33(\mathrm{CI}=1.93)$. The ORs for observed confounding variables in these models ranged between 0.65 and 2.07. For associations between low grip strength (vs. high) with depression, the $e$ value was $2.17(\mathrm{CI}=2)$, with anxiety it was $2.1(\mathrm{CI}=1.89)$, and for depression or anxiety, it was $2.11(\mathrm{CI}=1.96)$. Observed confounding variables in these models had ORs ranging from 0.64 to 2.16. For low combined fitness (vs. high), the $e$ value was 3.38 $(\mathrm{CI}=2.48)$ for depression, $2.49(\mathrm{CI}=1.56)$ for anxiety, and $3.03(\mathrm{CI}=2.28)$ for depression or anxiety. Observed confounding variables in these models had ORs ranging from 0.60 to 2.06 .

\section{Discussion}

\section{Main findings}

To the best of our knowledge, this is the first prospective study to examine associations between individual and combined CRF and grip strength with the incidence of common mental disorders in the general population. Low combined fitness (low CRF and low grip strength) was associated with 1.8 times the odds of a common mental disorder compared to high combined fitness, with the medium combined fitness group having 1.3 times the odds. Low combined fitness was associated with 2.0 and 1.6 times higher the odds of depression and anxiety disorders, respectively. When looking at CRF and grip strength as separate exposures, the effect sizes were smaller. We found that compared with high CRF, low CRF was associated with 1.5 times higher odds of a common mental disorder incidence and low grip strength with 1.4 times higher odds. There was some evidence of a dose-response relationship between fitness and the incidence of common mental disorders. We also found that associations with grip strength and anxiety disorders had higher odds ratios for women than men, and for older than younger adults.

These findings were robust to a series of sensitivity analyses. The findings are unlikely to be nullified by an unmeasured confounding variable according to the $e$ values. For example, the $e$ value for combined fitness groups indicates an unmeasured confounding variable would require an association of at least $\mathrm{OR}=3.0$ with fitness and common mental disorders to nullify the observed associations. The observed confounding variables had ORs ranging from 0.6 to 2.1, suggesting an OR of 3 for an unmeasured confounding variable is unlikely. Our results were also consistent in a full cohort with imputed missing data.

Our study is novel in demonstrating that the combination of low CRF and grip strength is associated with a higher risk of common mental disorders than either type of fitness alone. This finding highlights the importance of focusing on multiple components of fitness and their 
associations with mental health. Findings for individual exposures align with results from recent meta-analyses of 4 studies that suggested low CRF was associated with an increased risk of common mental disorders of similar effect size ( $\mathrm{HR}=1.47,95 \% \mathrm{CI} 1.23$ to 1.76$)$, which also suggested a dose-response relationship [28]. These findings build on previous prospective studies in smaller samples [34-37] to suggest that low grip strength is a possible risk factor for common mental disorders in adult men and women.

\section{Strengths and limitations}

This study benefitted from a large sample size and a 7year follow-up period. It included objective measures of fitness administered by trained staff using validated protocols. The prospective study design, dose-response relationship, consistent results from several sensitivity analyses (including the removal of participants with a history of depression or anxiety), and multiple imputation models, suggest possible biases such as attrition bias, reverse causation, or unmeasured confounding are unlikely to explain our results. The use of DAGs to inform our analysis also improved our ability to estimate causal effects.

The study also had several limitations. We estimated CRF from a submaximal exercise test rather than a gold standard maximal exercise with gas analysis [57]. However, these tests are prohibitively expensive in large samples and we are aware of only one previous study $(n=$ 1575 ) in this area using these gold standard exercise protocols [58]. Exercise testing was a late addition to Biobank data collection protocols in 2009 and only available for $\sim 14 \%$ of participants. However, these participants are representative of the wider Biobank sample in terms of sociodemographic and biological characteristics [47]. Despite the low response rate of $5.5 \%$ in obtaining the final Biobank sample, participants are comparable with the general population on several sociodemographic and physical health factors $[44,59]$.

The observational nature of this study also includes the risk of unmeasured confounding biasing our results. The $e$ values suggest it is unlikely that a single unmeasured confounding variable would explain our main findings. However, multiple unmeasured variables together might explain the associations we found. There may also be some measurement error in the self-reported scales used to record common mental health symptoms compared with clinical diagnoses. However, these scales allowed us to assess symptoms in people who do not seek treatment or are without a formal mental health diagnosis and are extensively validated. It was also not possible to reliably estimate common mental disorder incidence at baseline, only symptoms.

\section{Implications and future research}

Aerobic and resistance training improves different aspects of physical fitness and randomised controlled trials have found that both types of training can each reduce common mental health symptoms [5-11]. At a population level, improving multiple aspects of fitness could contribute towards a reduction in the incidence of common mental disorders. While broadly increasing physical activity will be beneficial $[12,13]$, structured aerobic and resistance exercises with sufficient intensity to improve fitness may have a greater effect on risk reduction. These combined approaches may also have additive benefits for reducing the physical health risks [39-42] associated with common mental disorders.

It is possible to modify fitness through simple, lowcost physical activity interventions, including in people with common mental health symptoms [60]. Substantial improvements in fitness are possible within a short timeframe. For example, one study in previously untrained older adults suggested that 3 weeks of regular aerobic exercise was sufficient to improve CRF by $31 \%$, which continued to increase with further training [61]. Our data suggest that a $31 \%$ increase would equate to moving from low to medium CRF and reduce the odds of a common mental disorder by $14.1 \%$. Similar improvements in grip strength over the same period would further reduce the odds of a common mental disorder by $32.5 \%$.

Objective markers of physical fitness approximate habitual physical activity and could also be useful population-level indicators of mental health risk in their own right. Most studies of associations between physical activity and the incidence of common mental disorders use self-reported activity data [12, 13]. Our study suggests that objective markers of physical fitness could be useful indicators of mental health risk in the population, as they are for physical health risks [20, 21]. Metaanalyses suggest that high physical activity is associated with a 0.83 (95\% CI, 0.79 to 0.88$)$ and 0.74 (95\% CI, 0.62 to 0.88$)[12,13]$ lower odds of a depression and anxiety disorders respectively. However, effect sizes for low (combined or individual) physical fitness appear larger in this study and previous meta-analyses [28]. Objective markers of fitness are increasingly recognised as stronger indicators of cardiovascular disease than physical activity $[25,62]$, and the same may be true for mental health.

The collection of population-level physical activity data will continue to play a foundational role in monitoring physical and mental health but remains challenging [63]. Physical fitness is a stable surrogate marker of habitual physical activity with objective, clearly defined outputs, such as oxygen uptake [18]. Fitness may also act through pathways independent of general physical activity $[64,65]$. For example, measures of CRF can incorporate additional information by capturing the 
complex interplay between other relevant factors to mental health, such as genetics, body composition, and smoking [66]. Increased efforts to collect populationlevel physical fitness data could inform our understanding of mental health and the development of public health strategies.

\section{Conclusions}

Low CRF and grip strength are both associated with an increased incidence of common mental disorders, and the combination of low CRF and low grip strength was associated with the highest level of risk. Physical fitness could be an objectively measurable indicator and a modifiable risk factor for common mental disorders in the population. Public health approaches to improve physical fitness through combined aerobic and resistance activities could reduce the incidence of common mental disorders and improve physical health outcomes for people with mental health symptoms.

\section{Supplementary information}

Supplementary information accompanies this paper at https://doi.org/10 1186/s12916-020-01782-9.

Additional file 1. Additional details on the methodology (Figure 1 and Methodology 1), baseline participant characteristics (Tables 1 and 2), sensitivity analyses results (Tables 3 to 6), and STROBE statement.

\section{Abbreviations}

CRF: Cardiorespiratory fitness

\section{Acknowledgements}

We are grateful to all participants and staff involved in UK Biobank. This research is a part of project identification number 51093.

\section{Authors' contributions}

AK, JH, BS, and DO conceptualised the study; AK analysed all the data. All authors wrote and edited the final manuscript. AK had full access to the UK Biobank data. All authors read and approved the final manuscript.

\section{Funding}

The UK Biobank is funded by the Wellcome Trust, Medical Research Council, Department of Health, Scottish government, Northwest Regional Development Agency, Welsh Assembly government, and British Heart Foundation.

Aaron Kandola is supported by the ESRC (ES/P000592/1). Brendon Stubbs is supported by a Clinical Lectureship (ICA-CL-2017-03-001) jointly funded by Health Education England (HEE) and the National Institute for Health Research (NIHR). Brendon Stubbs is part funded by the NIHR Biomedical Research Centre at South London and Maudsley NHS Foundation Trust. Brendon Stubbs also holds active grants with the Medical Research Council and Guys and St Thomas Charity (GSTT). Brendon Stubbs has received consultancy fees from ASICS Europe BV. Joseph Hayes is supported by the Wellcome Trust (211085/Z/18/Z). David Osborn and Joseph Hayes are supported by the UCLH NIHR Biomedical Research Centre and are also part supported by the NIHR Collaboration for Leadership in Applied Health Research and Care North Thames at Bart's Health NHS Trust. No funding body had any influence on the conception, design, analysis, or production of this manuscript, and the views it expresses are not necessarily those of any funding organisations.

\section{Availability of data and materials}

All bona fide researchers can apply to access and use the UK Biobank resource.

\section{Ethics approval and consent to participate}

The authors assert that all procedures contributing to this work comply with the ethical standards of the relevant national and institutional committees on human experimentation and with the Helsinki Declaration of 1975, as revised in 2008. All procedures involving human subjects/patients were approved for the UK Biobank from the North West Multi-centre Research Ethics Committee (11/NW/03820). All UK Biobank participants gave informed consent in accordance with the Declaration of Helsinki prior to enrolment in the study.

\section{Consent for publication}

Not applicable

\section{Competing interests}

The authors declare that they have no competing interests.

\section{Author details}

${ }^{1}$ Division of Psychiatry, University College London, Maple House, 149 Tottenham Court Rd, London W1T 7BN, UK. ${ }^{2}$ Camden and Islington NHS Foundation Trust, London, UK. ${ }^{3}$ Department of Psychological Medicine, Institute of Psychiatry, Psychology, and Neuroscience, King's College London, London, UK. ${ }^{4}$ Physiotherapy Department, South London, and Maudsley National Health Services Foundation Trust, London, UK. ${ }^{5}$ Department of Psychiatry, Massachusetts General Hospital, Boston, MA, USA. ${ }^{6}$ Department of Epidemiology, Harvard T.H. Chan School of Public Health, Boston, MA, USA. ${ }^{7}$ Psychiatric \& Neurodevelopmental Genetics Unit, Center for Genomic Medicine, Massachusetts General Hospital, Boston, MA, USA. ${ }^{8}$ Stanley Center for Psychiatric Research, Broad Institute, Boston, MA, USA.

Received: 29 July 2020 Accepted: 15 September 2020

Published online: 11 November 2020

References

1. World Health Organisation. Depression and other common mental disorders global health estimates. Geneva: World Health Organization; 2017.

2. Batelaan NM, Seldenrijk A, Bot M, van Balkom AJLM, Penninx BWJH. Anxiety and new onset of cardiovascular disease: critical review and meta-analysis. Br J Psychiatry. 2016;208(03):223-31.

3. Machado MO, Veronese N, Sanches M, Stubbs B, Koyanagi A, Thompson T, et al. The association of depression and all-cause and cause-specific mortality: an umbrella review of systematic reviews and meta-analyses. BMC Med. 2018;16(1):112.

4. Walker ER, RE MG, Druss BG. Mortality in Mental disorders and global disease burden implications. JAMA Psychiatry. 2015;72(4):334.

5. Bridle C, Spanjers K, Patel S, Atherton NM, Lamb SE. Effect of exercise on depression severity in older people: systematic review and meta-analysis of randomised controlled trials. Br J Psychiatry. 2012;201 (3):180-5.

6. Cooney GM, Dwan K, Greig CA, Lawlor DA, Rimer J, Waugh FR, et al. Exercise for depression. Cochrane Database Syst Rev. 2013;(9):1465-1858.

7. Josefsson T, Lindwall M, Archer T. Physical exercise intervention in depressive disorders: meta-analysis and systematic review. Scand J Med Sci Sports. 2014;24(2):259-72.

8. Kvam S, Kleppe CL, Nordhus $\mathrm{H}$, Hovland A. Exercise as a treatment for depression: a meta-analysis. J Affect Disord. 2016;202:67-86.

9. Schuch FB, Vancampfort D, Richards J, Rosenbaum S, Ward PB, Stubbs B. Exercise as a treatment for depression: a meta-analysis adjusting for publication bias. J Psychiatr Res. 2016;77:42-51.

10. Herring MP, Puetz TW, O'Connor PJ, Dishman RK. Effect of exercise training on depressive symptoms among patients with a chronic illness. Arch Intern Med. 2012;172(2):101.

11. Stubbs B, Vancampfort D, Rosenbaum S, Firth J, Cosco T, Veronese N, et al. An examination of the anxiolytic effects of exercise for people with anxiety and stress-related disorders: a meta-analysis. Psychiatry Res. 2017;249:102-8.

12. Schuch FB, Vancampfort D, Firth J, Rosenbaum Ward PB, Silva E, Hallgren Dunn AL, Deslandes Fleck MC, Carvalho AF, Stubbs B, Schuch FB, Vancampfort D, Firth J, Rosenbaum S, Ward PB, et al. Physical activity and 
incident depression: a meta-analysis of prospective cohort studies. Am J Psychiatry. 2018;(in press)(7):631-48.

13. Schuch FB, Stubbs B, Meyer J, Heissel A, Zech P, Vancampfort D, Carvalho AF. Physical activity protects from incident anxiety: A meta-analysis of prospective cohort studies. Depression and anxiety. 2019;36(9):846-58.

14. McDowell CP, Dishman RK, Gordon BR, Herring MP. Physical activity and anxiety: a systematic review and meta-analysis of prospective cohort studies. Am J Prev Med. 2019;57(4):545-56.

15. Teychenne M, Costigan SA, Parker $\mathrm{K}$. The association between sedentary behaviour and risk of anxiety: a systematic review. BMC Public Health. 2015; 15(1):513.

16. Teychenne M, Ball K, Salmon J. Sedentary behavior and depression among adults: a review. Int J Behav Med. 2010;17(4):246-54.

17. Prince SA, Adamo KB, Hamel M, Hardt J, Connor Gorber S, Tremblay M. A comparison of direct versus self-report measures for assessing physical activity in adults: a systematic review. Int J Behav Nutr Phys Act. 2008;5(1):56.

18. Carrick-Ranson G, Hastings JL, Bhella PS, Fujimoto N, Shibata S, Palmer MD, et al. The effect of lifelong exercise dose on cardiovascular function during exercise. J Appl Physiol. 2014;116(7):736-45.

19. Church TS, Earnest CP, Skinner JS, Blair SN. Effects of different doses of physical activity oncardiorespiratory fitness among sedentary, overweight or obese postmenopausal women with elevated blood pressure: a randomized controlled trial. J Am Med Assoc. 2007;297(19):2081-91.

20. Cooper R, Kuh D, Hardy R. Objectively measured physical capability levels and mortality: systematic review and meta-analysis. BMJ. 2010;341:639.

21. Ortega FB, Ruiz JR, Castillo MJ, Sjöström M. Physical fitness in childhood and adolescence: a powerful marker of health. Int J Obes. 2008;32:1-11.

22. Kodama S, Saito K, Tanaka S, Maki M, Yachi Y, Asumi M, et al. Cardiorespiratory fitness as a quantitative predictor of all-cause mortality and cardiovascular events in healthy men and women. JAMA. 2009;301(19):2024.

23. Lee D, Sui X, Artero EG, Lee I-M, Church TS, McAuley PA, et al. Long-term effects of changes in cardiorespiratory fitness and body mass index on all-cause and cardiovascular disease mortality in men. Circulation. 2011;124(23):2483-90.

24. Blair SN, Kampert JB, Kohl HW, Barlow CE, Macera CA, Paffenbarger RS, et al. Influences of cardiorespiratory fitness and other precursors on cardiovascular disease and all-cause mortality in men and women. JAMA. 1996;276(3):205.

25. Myers J, Kokkinos P, Chan K, Dandekar E, Yilmaz B, Nagare A, et al. Cardiorespiratory fitness and reclassification of risk for incidence of heart failure. Circ Hear Fail. 2017;10(6):e003780.

26. Volaklis KA, Halle M, Meisinger C. Muscular strength as a strong predictor of mortality: a narrative review. Eu J Intern Med. 2015;26:303-10.

27. Wu Y, Wang W, Liu T, Zhang D. Association of grip strength with risk of allcause mortality, cardiovascular diseases, and cancer in community-dwelling populations: a meta-analysis of prospective cohort studies. J Am Med Dir Assoc. 2017;18(6):551.e17-35.

28. Kandola A, Ashdown-Franks G, Stubbs B, Osborn DPJ, Hayes JF. The association between cardiorespiratory fitness and the incidence of common mental health disorders: a systematic review and meta-analysis. J Affect Disord. 2019;257:748-57.

29. Roberts HC, Denison HJ, Martin HJ, Patel HP, Syddall H, Cooper C, et al. A review of the measurement of grip strength in clinical and epidemiological studies: towards a standardised approach. 2011;

30. Wind AE, Takken T, Helders PJM, Engelbert RHH. Is grip strength a predictor for total muscle strength in healthy children, adolescents, and young adults? Eur J Pediatr. 2010;169(3):281-7.

31. Aadahl M, Beyer N, Linneberg A, Thuesen BH, Jørgensen T. Grip strength and lower limb extension power in 19-72-year-old Danish men and women: The Health 2006 study. BMJ Open. 2011;1(2):e000192.

32. Ashdown-Franks G, Stubbs B, Koyanagi A, Schuch F, Firth J, Veronese N, et al. Handgrip strength and depression among 34,129 adults aged 50 years and older in six low- and middle-income countries. J Affect Disord. 2019; 243:448-54.

33. Han KM, Chang J, Yoon HK, Ko YH, Ham BJ, Kim YK, et al. Relationships between hand-grip strength, socioeconomic status, and depressive symptoms in community-dwelling older adults. J Affect Disord. 2019;252: 263-70.

34. Veronese N, Stubbs B, Trevisan C, Bolzetta F, De Rui M, Solmi M, et al. Poor physical performance predicts future onset of depression in elderly people: Progetto Veneto Anziani Longitudinal Study. Phys Ther. 2017;97(6):659-68.
35. Hamer M, Batty GD, Kivimaki M. Sarcopenic obesity and risk of new onset depressive symptoms in older adults: English Longitudinal Study of Ageing. Int J Obes. 2015;39(12):1717-20.

36. McDowell CP, Gordon BR, Herring MP. Sex-related differences in the association between grip strength and depression: results from the Irish Longitudinal Study on Ageing. Exp Gerontol. 2018;104:147-52.

37. Gordon BR, McDowell CP, Lyons M, Herring MP. Associations between grip strength and generalized anxiety disorder in older adults: results from the Irish longitudinal study on ageing. J Affect Disord. 2019;255:136-41.

38. Kessler RC, Gruber M, Hettema JM, Hwang I, Sampson N, Yonkers KA. Comorbid major depression and generalized anxiety disorders in the National Comorbidity Survey follow-up. Psychol Med. 2008;38(03):365-74.

39. Church TS, Blair SN, Cocreham S, Johannsen N, Johnson W, Kramer K, et al. Effects of aerobic and resistance training on hemoglobin A1c levels in patients with type 2 diabetes: a randomized controlled trial. JAMA. 2010; 304(20):2253-62.

40. AbouAssi H, Slentz CA, Mikus CR, Tanner CJ, Bateman LA, Willis LH, et al, The effects of aerobic, resistance, and combination training on insulin sensitivity and secretion in overweight adults from STRRIDE AT/RT: a randomized trial. J Appl Physiol. 2015;118(12):1474-82.

41. Ho SS, Dhaliwal SS, Hills AP, Pal S. The effect of 12 weeks of aerobic, resistance or combination exercise training on cardiovascular risk factors in the overweight and obese in a randomized trial. BMC Public Health. 2012; 12(1):704.

42. Villareal DT, Aguirre L, Gurney AB, Waters DL, Sinacore DR, Colombo E, et al. Aerobic or resistance exercise, or both, in dieting obese older adults. N Engl J Med. 2017;376(20):1943-55.

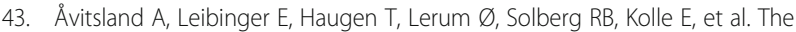
association between physical fitness and mental health in Norwegian adolescents. BMC Public Health. 2020;20(1):776.

44. Collins R. What makes UK Biobank special? Lancet. 2012;379(9822):1173-4.

45. Sudlow C, Gallacher J, Allen N, Beral V, Burton P, Danesh J, et al. UK Biobank: an open access resource for identifying the causes of a wide range of complex diseases of middle and old age. PLoS Med. 2015;12(3):e1001779.

46. Celis-Morales CA, Welsh P, Lyall DM, Steell L, Petermann F, Anderson J, et al. Associations of grip strength with cardiovascular, respiratory, and cancer outcomes and all cause mortality: prospective cohort study of half a million UK Biobank participants. BMJ. 2018:8:361.

47. Celis-Morales CA, Lyall DM, Anderson J, lliodromiti S, Fan Y, Ntuk UE, Gill JM. The association between physical activity and risk of mortality is modulated by grip strength and cardiorespiratory fitness: evidence from 498135 UKBiobank participants. Eur Heart J. 2017;38(2):116-22.

48. Tanaka H, Monahan KD, Seals DR. Age-predicted maximal heart rate revisited. J Am Coll Cardiol. 2001;37(1):153-6.

49. Swain DP. Energy cost calculations for exercise prescription: An update. Sports Med. 2000;30:17-22.

50. Kroenke K, Spitzer RL, Williams JBW. The PHQ-9: validity of a brief depression severity measure. J Gen Intern Med. 2001;16(9):606-13.

51. Löwe B, Wahl I, Rose M, Spitzer C, Glaesmer H, Wingenfeld K, et al. A 4-item measure of depression and anxiety: validation and standardization of the Patient Health Questionnaire-4 (PHQ-4) in the general population. J Affect Disord. 2010;122(1-2):86-95.

52. Spitzer RL, Kroenke K, Williams JBW, Löwe B. A brief measure for assessing generalized anxiety disorder: the GAD-7. Arch Intern Med. 2006;166(10): 1092-7.

53. Moriarty AS, Gilbody S, McMillan D, Manea L. Screening and case finding for major depressive disorder using the Patient Health Questionnaire (PHQ-9): a meta-analysis. Gen Hosp Psychiatry. 2015;37(6):567-76.

54. Plummer F, Manea L, Trepel D, McMillan D. Screening for anxiety disorders with the GAD-7 and GAD-2: a systematic review and diagnostic metaanalysis. Gen Hosp Psychiatry. 2016;39:24-31.

55. Haneuse S, VanderWeele TJ, Arterburn D. Using the E-value to assess the potential effect of unmeasured confounding in observational studies. JAMA. 2019:321(6):602-3

56. VanderWeele TJ, Ding P. Sensitivity analysis in observational research: introducing the E-value. Ann Intern Med. 2017;167(4):268-74.

57. Medicine AC of S. ACSM's guidelines for exercise testing and prescription. Baltimore: Lippincott Williams \& Wilkins; 2013.

58. Baumeister SE, Leitzmann MF, Bahls M, Dörr M, Schmid D, Schomerus G, et al. Associations of leisure-time and occupational physical activity and cardiorespiratory fitness with incident and recurrent major depressive 
disorder, depressive symptoms, and incident anxiety in a general population. J Clin Psychiatry. 2017;78(01):e41-7.

59. Hamer M, Patalay P, Bell S, Batty GD. Change in device-measured physical activity assessed in childhood and adolescence in relation to depressive symptoms: a general population-based cohort study. J Epidemiol Community Health. 2020;74(4):330-5.

60. Stubbs B, Rosenbaum S, Vancampfort D, Ward PB, Schuch FB. Exercise improves cardiorespiratory fitness in people with depression: a metaanalysis of randomized control trials. J Affect Disord. 2016;190:249-53.

61. Murias JM, Kowalchuk JM, Paterson DH. Time course and mechanisms of adaptations in cardiorespiratory fitness with endurance training in older and young men. J Appl Physiol. 2010;108(3):621-7.

62. Swift DL, Lavie CJ, Johannsen NM, Arena R, Earnest CP, O'Keefe JH, et al. Physical activity, cardiorespiratory fitness, and exercise training in primary and secondary coronary prevention. Circ J. 2013;77(2):281-92.

63. Ainsworth B, Cahalin L, Buman M, Ross R. The current state of physical activity assessment tools. Prog Cardiovasc Dis. 2015;57(4):387-95.

64. Bouchard C, Sarzynski MA, Rice TK, Kraus WE, Church TS, Sung YJ, et al. Genomic predictors of the maximal $\mathrm{O}_{2}$ uptake response to standardized exercise training programs. J Appl Physiol. 2011;110(5):1160-70.

65. Frederiksen H, Gaist D, Christian Petersen H, Hjelmborg J, McGue M, Vaupel JW, et al. Hand grip strength: a phenotype suitable for identifying genetic variants affecting mid- and late-life physical functioning. Genet Epidemiol. 2002;23(2):110-22

66. DeFina LF, Haskell WL, Willis BL, Barlow CE, Finley CE, Levine BD, et al. Physical activity versus cardiorespiratory fitness: two (partly) distinct components of cardiovascular health? Prog Cardiovasc Dis. 2015;57(4):324-9.

\section{Publisher's Note}

Springer Nature remains neutral with regard to jurisdictional claims in published maps and institutional affiliations.

Ready to submit your research? Choose BMC and benefit from:

- fast, convenient online submission

- thorough peer review by experienced researchers in your field

- rapid publication on acceptance

- support for research data, including large and complex data types

- gold Open Access which fosters wider collaboration and increased citations

- maximum visibility for your research: over $100 \mathrm{M}$ website views per year

At BMC, research is always in progress.

Learn more biomedcentral.com/submissions 